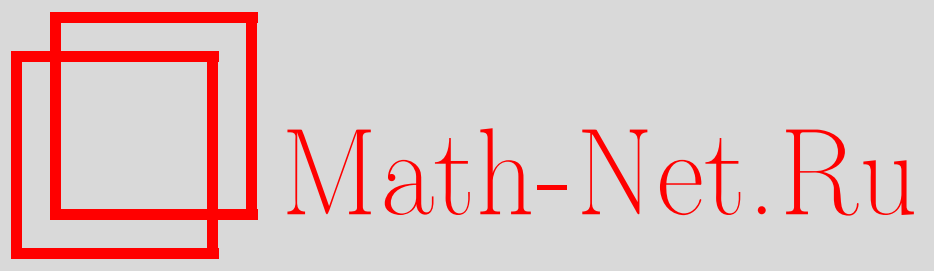

С. Д. Глызин, А. Ю. Колесов, Н. Х. Розов, Об одном подходе к моделированию искусственных генных сетей, ТМФ, 2018, том 194, номер 3, 547-568

DOI: https://doi.org/10.4213/tmf9321

Использование Общероссийского математического портала Math-Net.Ru подразумевает, что вы прочитали и согласны с пользовательским соглашением http://www . mathnet.ru/rus/agreement

Параметры загрузки:

IP: 3.85 .73 .92

26 апреля 2023 г., 14:59:54

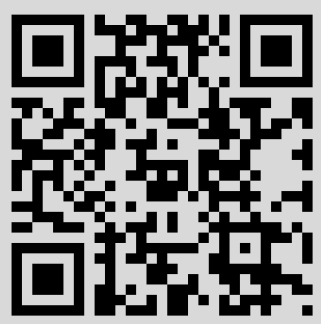




\title{
ОБ ОДНОМ ПОДХОДЕ К МОДЕЛИРОВАНИЮ ИСКУССТВЕННЫХ ГЕННЫХ СЕТЕЙ
}

\begin{abstract}
Предлагается новая математическая модель репрессилятора - простейшей кольцевой генной сети, состоящей из трех элементов. Рассматриваемая модель представляет собой некоторую трехмерную систему обыкновенных дифференциальных уравнений, зависящую от одного параметра. Исследуются вопросы о существовании и устойчивости в этой системе релаксационного периодического движения.
\end{abstract}

Ключевые слова: репрессилятор, генные сети, релаксационный цикл, асимптотика, устойчивость.

DOI: https://doi.org/10.4213/tmf9321

\section{1. ПОСТАНОВКА ПРОБЛЕМЫ И ОПИСАНИЕ РЕЗУЛЬТАТА}

Интерес к искусственным генетическим осцилляторам вызван тем обстоятельством, что они являются упрощенными моделями таких ключевых биологических процессов, как клеточный цикл и циркадные ритмы. Простейший генетический осциллятор, предложенный в работе [1] и названный репрессилятором, состоит из трех элементов. Каждый из этих элементов однонаправленно ингибирует соседний (см. рис. 1, на котором элемент $A$ подавляет синтез $B$, элемент $B$ подавляет синтез $C$, а третий элемент $C$, замыкая цикл, подавляет синтез $A$ ).

Математическая модель системы, показанной на рис. 1, имеет вид

$$
\dot{m}_{j}=-m_{j}+\frac{\alpha}{1+u_{j-1}^{\gamma}}+\alpha_{0}, \quad \dot{u}_{j}=\beta\left(m_{j}-u_{j}\right), \quad j=1,2,3
$$

Работа выполнена в рамках государственного задания Министерства образования и науки РФ (проект № 1.10160.2017/5.1) и при поддержке РФФИ (грант № 15-01-04066_a).

* Ярославский государственный университет им. П. Г. Демидова, Ярославль, Россия. E-mail: glyzin@uniyar.ac.ru, kolesov@uniyar.ac.ru

${ }^{\dagger}$ Московский государственный университет им. М. В. Ломоносова, Москва, Россия. E-mail: fpo.mgu@mail.ru 


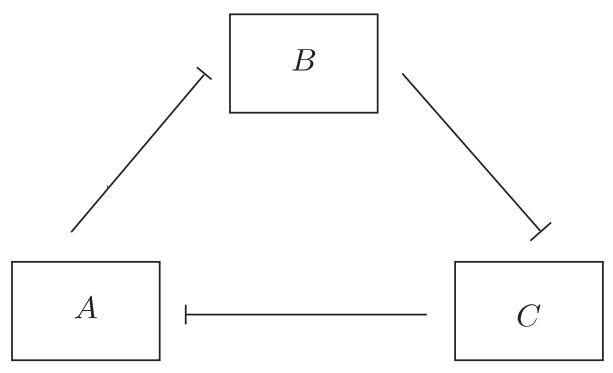

Рис. 1

где $u_{0}=u_{3}$. Следуя работе [1], считаем, что каждый элемент осциллятора представляет собой набор из мРНК (матричной рибонуклеиновой кислоты) с концентрацией $m_{j}$ и белка с концентрацией $u_{j}$. Предполагаем далее, что изменение во времени концентраций $m_{j}$ характеризуется процессами синтеза и деградации. Первый из этих процессов описывается функцией $\alpha /\left(1+u_{j-1}^{\gamma}\right)$, где $u_{j-1}-$ концентрация белка-репрессора для $j$-й мРНK, $\gamma=$ const $>0$ - коэффициент кооперативности, $\alpha=$ const $>0$ - скорость транскрипции в отсутствие репрессора. Второй процесс описывается линейным слагаемым " $-m_{j}$ ". И, наконец, аддитивная добавка $\alpha_{0}>0$ в уравнении для $m_{j}$ отвечает за утечку промотора.

В случае концентраций белков $u_{j}$ ситуация проще. А именно, предполагаем, что их динамика представлена линейными процессами синтеза (слагаемое $\beta m_{j}$ в уравнении для $u_{j}$ из системы (1.1)) и деградации (слагаемое "- $\beta u_{j}$ " в том же уравнении). Здесь $\beta=$ const $>0$ - отношение скорости деградации белка к скорости деградации мРНK.

Как правило, исследование модели (1.1) проводится в предположении о малости $\beta$ и $\alpha_{0}$. В этой ситуации после замены $\beta t \rightarrow t$ и отбрасывания добавки $\alpha_{0}$ получается сингулярно возмущенная система, к которой затем применяется известный принцип сведения Тихонова [2]. Результатом этого сведения оказывается система

$$
\dot{u}_{j}=-u_{j}+\frac{\alpha}{1+u_{j-1}^{\gamma}}, \quad j=1,2,3, \quad u_{0}=u_{3} .
$$

Вопрос об автоколебаниях системы (1.2) и аналогичных систем, возникающих при моделировании генных сетей, изучался многими авторами (см., например, [3]-[8]). Из содержащихся в указанных работах результатов аналитических и численных исследований вытекает существование у модели (1.2) при $\gamma>2$ и при подходящем увеличении $\alpha$ устойчивого цикла, обладающего свойством самосимметричности (инвариантности по отношению к циклическим перестановкам координат). Из упомянутого свойства следует, что этот цикл допускает представление вида

$$
\left(u_{1}, u_{2}, u_{3}\right)=(u(t), u(t+h), u(t+2 h)),
$$

где $h>0-$ фазовый сдвиг, и имеет период $T=3 h$.

Описанный выше характер взаимодействия концентраций $u_{j}$ и $m_{j}$ вполне аналогичен взаимодействию шести экологических популяций - трех хищников и трех жертв. Действительно, предположим, что $u_{j}, j=1,2,3$, и $m_{j}, j=1,2,3,-$ плотности численности популяций хищников и жертв соответственно. Тогда в силу (1.1) 
каждый хищник $u_{j}$ питается только одной жертвой $m_{j}$ (при $m_{j} \equiv 0$ численность $u_{j}$ затухает по экспоненциальному закону) и в то же время оказывает давление только на жертву $m_{j+1}$. Последнее выражается в том, что при увеличении $u_{j}$ уменьшается скорость роста численности $m_{j+1}$. Кроме того, при отсутствии хищника-репрессора $\left(u_{j-1} \equiv 0\right)$ численность $m_{j}$ стремится при $t \rightarrow+\infty$ к пороговому значению $m_{j}=$ $\alpha+\alpha_{0}$.

Для математического моделирования интересующей нас генной сети приведенная экологическая интерпретация позволяет воспользоваться подходом Колесова [9]. Упомянутый подход приводит к системе

$$
\dot{m}_{j}=\frac{r_{1}}{1+a}\left[1+a\left(1-u_{j-1}\right)-m_{j}\right] m_{j}+\alpha, \quad \dot{u}_{j}=r_{2}\left[m_{j}-u_{j}\right] u_{j}, \quad j=1,2,3,
$$

где $u_{0}=u_{3}$ и все постоянные $r_{1}, r_{2}, a, \alpha$ положительны. Подчеркнем, что в уравнение для $m_{j}$, нарушая его вольтерровскую структуру, мы специально добавили последнее слагаемое $\alpha$, аналогичное последнему слагаемому $\alpha_{0}$ в (1.1). Как будет показано ниже, в отличие от системы (1.1), где можно положить $\alpha_{0}=0$, в нашем случае требование $\alpha>0$ является обязательным.

Как и система (1.1), новая математическая модель репрессилятора (1.4) допускает некоторое упрощение. А именно, предположим сначала, что $r_{2} \gg 1, r_{1}=r \sim 1$. Тогда согласно принципу сведения [2] при $r_{2} \rightarrow+\infty$ имеем $m_{j}=u_{j}, j=1,2,3$. Что же касается компонент $u_{j}$, то для них получаем систему

$$
\dot{u}_{j}=\frac{r}{1+a}\left[1+a\left(1-u_{j-1}\right)-u_{j}\right] u_{j}+\alpha, \quad j=1,2,3, \quad u_{0}=u_{3} .
$$

На следующем этапе упрощения устремим в (1.5) параметр $a \mathrm{~K}+\infty$ и в получившейся системе выполним замены $r t \rightarrow t, \alpha / r \rightarrow \alpha$. В результате приходим к аналогичной (1.2) трехмерной модели репрессилятора

$$
\dot{u}_{j}=\left(1-u_{j-1}\right) u_{j}+\alpha, \quad j=1,2,3, \quad u_{0}=u_{3} .
$$

Локальный и численный анализ системы (1.6) приводит к выводу, что ее динамика по параметру $\alpha$ достаточно проста. А именно, в инвариантном конусе $K \subset \mathbb{R}^{3}$ векторов с положительными координатами она имеет единственное состояние равновесия

$$
u_{1}=u_{2}=u_{3}=u_{*}, \quad u_{*}=\frac{1+\sqrt{1+4 \alpha}}{2},
$$

экспоненциально устойчивое при $\alpha>2$ и неустойчивое при $0<\alpha<2$. Далее, при уменьшении параметра $\alpha$ и при прохождении его через критическое значение $\alpha=2$ в результате бифуркации Андронова-Хопфа от состояния равновесия (1.7) ответвляется устойчивый цикл вида (1.3). Этот цикл сохраняется при всех $\alpha \in(0,2)$, а при $\alpha \rightarrow 0$ приобретает релаксационный характер. Наглядное представление о нем дает график зависимости от $t$ фигурирующей в (1.3) функции $u(t)$ при $\alpha=0.05$ (см. рис. 2).

В настоящей статье рассматриваются вопросы о существовании, асимптотике и устойчивости цикла (1.3) системы (1.6) при $\alpha \ll 1$. Точнее говоря, анализу подлежит система

$$
\dot{x}=\left(1-\frac{z}{\varepsilon}\right) x+\varepsilon^{2}, \quad \dot{y}=\left(1-\frac{x}{\varepsilon}\right) y+\varepsilon^{2}, \quad \dot{z}=\left(1-\frac{y}{\varepsilon}\right) z+\varepsilon^{2},
$$




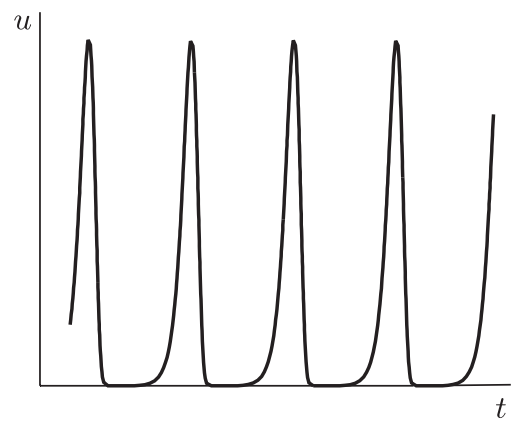

Рис. 2

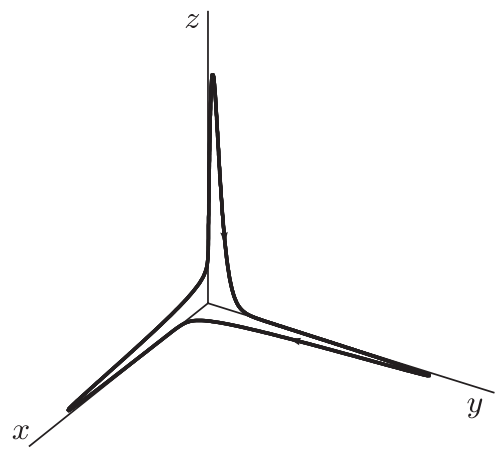

Рис. 3

получающаяся из (1.6) при заменах $u_{1}=x / \alpha, u_{2}=y / \alpha, u_{3}=z / \alpha$ и при $\alpha=\varepsilon \ll 1$. Для этой системы ниже будет установлено следующее утверждение.

Теорема 1. Найдется такое достаточно малое положительное $\varepsilon_{0}$, что при всех $0<\varepsilon \leqslant \varepsilon_{0}$ система (1.8) допускает экспоненииально орбитально устойчивый самосимметричный иикл

$$
C:(x, y, z)=\left(x_{*}(t, \varepsilon), x_{*}(t+h(\varepsilon), \varepsilon), x_{*}(t+2 h(\varepsilon), \varepsilon)\right) \in K
$$

периода $T=3 h(\varepsilon)$. Кроме того, для функиии $x_{*}(t, \varepsilon), x_{*}(0, \varepsilon) \equiv 1$, и фазового сдвига $h(\varepsilon)$ при $\varepsilon \rightarrow 0$ справедливы асимптотические представления

$$
\begin{aligned}
\max _{0 \leqslant t \leqslant T} x_{*}(t, \varepsilon) & =\left(1+O\left(\frac{\ln \ln (1 / \varepsilon)}{\ln (1 / \varepsilon)}\right)\right) e^{-1} \ln \frac{1}{\varepsilon} \\
\min _{0 \leqslant t \leqslant T} x_{*}(t, \varepsilon) & =\left(1+O\left(\frac{\ln \ln (1 / \varepsilon)}{\ln (1 / \varepsilon)}\right)\right) \frac{\varepsilon^{3}}{\ln (1 / \varepsilon)} e \\
h(\varepsilon) & =\ln \frac{1}{\varepsilon}+\ln \ln \frac{1}{\varepsilon}+O\left(\frac{\ln \ln (1 / \varepsilon)}{\ln (1 / \varepsilon)}\right) .
\end{aligned}
$$

Наглядное представление о цикле (1.9) дает рис. 3, где изображена его фазовая траектория при $\varepsilon=0.05$. 


\section{2. ОСНОВНАЯ КОНСТРУКЦИЯ}

Ниже будет изложено геометрическое доказательство теоремы 1, основанное на асимптотическом анализе оператора последования Пуанкаре по траекториям системы (1.8). В связи с этим предпримем некоторые вспомогательные построения.

Основная особенность цикла системы (1.8) заключается в том, что в любой момент времени $t$ только одна его компонента имеет порядок единицы или асимптотически велика, а две остальные асимптотически малы. Принимая во внимание это обстоятельство, для произвольного вектора $w=\left(y_{0}, z_{0}\right) \in \mathbb{R}^{2}$, принадлежащего множеству

$$
\mathcal{U}=\left\{w=\left(y_{0}, z_{0}\right): y_{0}>0, z_{0}>0\right\},
$$

рассмотрим решение $(x(t, w, \varepsilon), y(t, w, \varepsilon), z(t, w, \varepsilon)), t \geqslant 0$, системы (1.8) с начальными условиями

$$
\left.x\right|_{t=0}=1,\left.\quad y\right|_{t=0}=\varepsilon^{3} y_{0},\left.\quad z\right|_{t=0}=\frac{\varepsilon}{\ln (1 / \varepsilon)} z_{0}
$$

(причина выбора таких порядков малости $y, z$ прояснится в последующем). Далее, обозначим через $t=h(w, \varepsilon)$ первый положительный корень уравнения

$$
z(t, w, \varepsilon)=1
$$

(если таковой существует) и определим вспомогательный оператор

$$
\left.w \rightarrow \Pi_{0}(w, \varepsilon) \stackrel{\text { def }}{=}\left(x(t, w, \varepsilon) \varepsilon^{-3}, y(t, w, \varepsilon) \varepsilon^{-1} \ln \frac{1}{\varepsilon}\right)\right|_{t=h(w, \varepsilon)} .
$$

Рассмотрим также вектор

$$
w_{*}=(1,1)
$$

из множества (2.1).

Подробный асимптотический анализ оператора (2.4) содержится в следующем разделе. Здесь же приведем итоговый результат.

ТЕОРема 2. По любому компактному множеству $\Omega \subset \mathcal{U}$ можно указать такое достаточно малое $\varepsilon_{0}=\varepsilon_{0}(\Omega)>0$, что при всех $0<\varepsilon \leqslant \varepsilon_{0}$ оператор (2.4) определен на $\Omega$ и удовлетворяет при $\varepsilon \rightarrow 0$ асимптотическому равенству

$$
\max _{w \in \Omega}\left(\left\|\Pi_{0}(w, \varepsilon)-w_{*}\right\|_{\mathbb{R}^{2}}+\left\|\partial_{w} \Pi_{0}(w, \varepsilon)\right\|_{\mathbb{R}^{2} \rightarrow \mathbb{R}^{2}}\right)=O\left(\frac{\ln \ln (1 / \varepsilon)}{\ln (1 / \varepsilon)}\right),
$$

где $\partial_{w}-$ производная Фреше по w.

Обратимся к доказательству теоремы 1 и заметим, что всю его техническую часть вбирает в себя теорема 2. Действительно, обозначим через П оператор последования Пуанкаре по траекториям системы (1.8), определенный на секущей плоскости $\{(x, y, z): x=1\}$ посредством соотношения

$$
\Pi:\left.\left(1, \varepsilon^{3} y_{0}, \frac{\varepsilon z_{0}}{\ln (1 / \varepsilon)}\right) \rightarrow(x(t, w, \varepsilon), y(t, w, \varepsilon), z(t, w, \varepsilon))\right|_{t=T(w, \varepsilon)},
$$




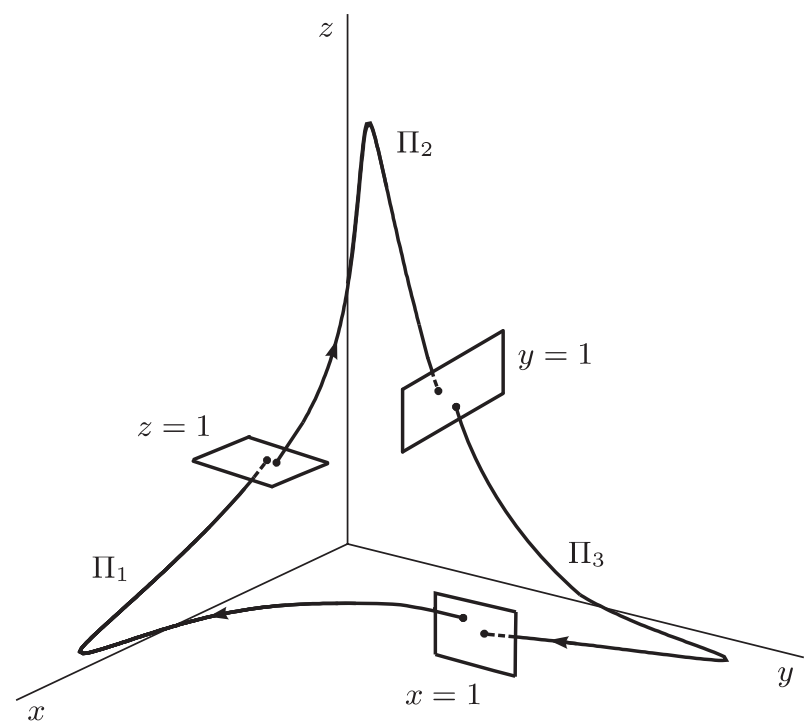

Рис. 4

где $t=T(w, \varepsilon)$ - второй положительный корень уравнения $x(t, w, \varepsilon)=1$, а $w$ принадлежит компактному множеству $\Omega \subset \mathcal{U}$. Будем считать это множество выбранным таким образом, что $w_{*} \in \operatorname{int} \Omega$, где $w_{*}-$ вектор $(2.5)$, int $\Omega$ - внутренность $\Omega$.

В свою очередь, оператор (2.7) можно представить в виде суперпозиции вида $\Pi_{3} \circ \Pi_{2} \circ \Pi_{1}$, где $\Pi_{1}$ - оператор соответствия по траекториям системы $(1.8)$, действующий из плоскости $\{(x, y, z): x=1\}$ в $\{(x, y, z): z=1\}$, а $\Pi_{2}, \Pi_{3}$ - аналогичные операторы, осуществляющие соответствие между плоскостями $\{(x, y, z): z=1\}$, $\{(x, y, z): y=1\}$ и $\{(x, y, z): y=1\},\{(x, y, z): x=1\}$ (см. рис. 4 , иллюстрирующий действия этих операторов). Из способа определения $\Pi_{0}$ (см. (2.4)) автоматически следует, что

$$
\Pi_{1}:\left(1, \varepsilon^{3} y_{0}, \frac{\varepsilon z_{0}}{\ln (1 / \varepsilon)}\right) \rightarrow\left(\varepsilon^{3} y_{(1)}, \frac{\varepsilon z_{(1)}}{\ln (1 / \varepsilon)}, 1\right),
$$

где $w_{(1)}=\left(y_{(1)}, z_{(1)}\right)=\Pi_{0}(w, \varepsilon)$. Далее, в силу $(2.6)$ имеем $w_{(1)}=w_{*}+o(1), \varepsilon \rightarrow 0$, и, следовательно, $w_{(1)} \in \Omega$ (так как, напомним, $w_{*} \in$ int $\Omega$ ).

При построении оператора $\Pi_{2}$ мы должны рассмотреть задачу Коши для системы (1.8) с начальными условиями

$$
\left.x\right|_{t=0}=\varepsilon^{3} y_{(1)},\left.\quad y\right|_{t=0}=\frac{\varepsilon}{\ln (1 / \varepsilon)} z_{(1)},\left.\quad z\right|_{t=0}=1 .
$$

Но поскольку задача (1.8), (2.9) после замены переменных $(\bar{x}, \bar{y}, \bar{z})=(z, x, y)$ и последующего переобозначения $(\bar{x}, \bar{y}, \bar{z}) \rightarrow(x, y, z)$ переходит в задачу вида $(1.8),(2.2)$ $\left(\right.$ с заменой $\left(y_{0}, z_{0}\right) \in \Omega$ на $\left.\left(y_{(1)}, z_{(1)}\right) \in \Omega\right)$, то для $\Pi_{2}$ получается аналогичное $(2.8)$ представление

$$
\Pi_{2}:\left(\varepsilon^{3} y_{(1)}, \frac{\varepsilon z_{(1)}}{\ln (1 / \varepsilon)}, 1\right) \rightarrow\left(\frac{\varepsilon z_{(2)}}{\ln (1 / \varepsilon)}, 1, \varepsilon^{3} y_{(2)}\right)
$$


где $w_{(2)}=\left(y_{(2)}, z_{(2)}\right)=\Pi_{0}\left(w_{(1)}, \varepsilon\right) \in \Omega$. Что же касается оператора $\Pi_{3}$, то для него из тех же соображений имеем

$$
\Pi_{3}:\left(\frac{\varepsilon z_{(2)}}{\ln (1 / \varepsilon)}, 1, \varepsilon^{3} y_{(2)}\right) \rightarrow\left(1, \varepsilon^{3} y_{(3)}, \frac{\varepsilon z_{(3)}}{\ln (1 / \varepsilon)}\right),
$$

где $w_{(3)}=\left(y_{(3)}, z_{(3)}\right)=\Pi_{0}\left(w_{(2)}, \varepsilon\right) \in \Omega$.

Возвращаясь к оператору $(2.7)$, заметим, что в координатах $\left(y_{0}, z_{0}\right)$ он записывается в виде

$$
\widetilde{\Pi}:\left.\left(y_{0}, z_{0}\right) \rightarrow\left(y(t, w, \varepsilon) \varepsilon^{-3}, z(t, w, \varepsilon) \varepsilon^{-1} \ln \frac{1}{\varepsilon}\right)\right|_{t=T(w, \varepsilon)} .
$$

В свою очередь, в силу соотношений (2.8), (2.10), (2.11) оператор (2.12) действует из $\Omega$ в $\Omega$ и задается равенством $\widetilde{\Pi}=\Pi_{0}^{3}$, где $\Pi_{0}$ - оператор (2.4). Остается добавить, что, во-первых, свойство (2.6) гарантирует существование у П 0 в множестве $\Omega$ единственной экспоненциально устойчивой неподвижной точки $w=w_{*}(\varepsilon), w_{*}(\varepsilon) \rightarrow w_{*}$ при $\varepsilon \rightarrow 0$, а во-вторых, в исходной системе (1.8) данной точке соответствует экспоненциально орбитально устойчивый цикл. А поскольку этот цикл является самосимметричным (плоскости $\{(x, y, z): x=1\},\{(x, y, z): z=1\}$ и $\{(x, y, z): y=1\}$ разбивают его на три участка, переходящих друг в друга при циклических перестановках координат), то он допускает представление (1.9), в котором

$$
x_{*}(t, \varepsilon)=\left.x(t, w, \varepsilon)\right|_{w=w_{*}(\varepsilon)}, \quad h(\varepsilon)=\left.h(w, \varepsilon)\right|_{w=w_{*}(\varepsilon)} .
$$

Итак, проблема доказательства теоремы 1 свелась к доказательству теоремы 2. В процессе обоснования последней для функций (2.13) будут получены требуемые асимптотические равенства (1.10).

\section{3. ДОКАЗАТЕЛЬСТВО ТЕОРЕМЫ 2}

Выберем произвольный компакт $\Omega \subset \mathcal{U}$ и обратимся к задаче Коши (1.8), (2.2) при $w=\left(y_{0}, z_{0}\right) \in \Omega$. Ниже будут получены равномерные по $w \in \Omega$ асимптотические формулы при $\varepsilon \rightarrow 0$ для компонент $x(t, w, \varepsilon), y(t, w, \varepsilon), z(t, w, \varepsilon)$ ее решения и их производных по $y_{0}, z_{0}$ на пяти различных промежутках изменения $t$.

На первом этапе рассмотрим отрезок времени

$$
0 \leqslant t \leqslant \bar{t}, \quad \bar{t}=\lambda_{1} \ln \ln \frac{1}{\varepsilon},
$$

где $\lambda_{1} \in(0,1)$ произвольно фиксировано. При указанных $t$ выполним в $(1.8)$ замены

$$
x=x_{1}, \quad y=\varepsilon^{3} y_{1}, \quad z=\frac{\varepsilon}{\ln (1 / \varepsilon)} z_{1} .
$$

В результате приходим к задаче Коши

$$
\begin{gathered}
\dot{x}_{1}=\left(1-\frac{z_{1}}{\ln (1 / \varepsilon)}\right) x_{1}+\varepsilon^{2}, \quad \varepsilon \dot{y}_{1}=\left(\varepsilon-x_{1}\right) y_{1}+1, \\
\dot{z}_{1}=\left(1-\varepsilon^{2} y_{1}\right) z_{1}+\varepsilon \ln \frac{1}{\varepsilon} \\
\left.x_{1}\right|_{t=0}=1,\left.\quad y_{1}\right|_{t=0}=y_{0},\left.\quad z_{1}\right|_{t=0}=z_{0} .
\end{gathered}
$$


Ее решение обозначим через $\left(x_{1}(t, w, \varepsilon), y_{1}(t, w, \varepsilon), z_{1}(t, w, \varepsilon)\right)$. Справедливо следующее утверждение.

ЛЕмма 1. На отрезке времени (3.1) для компонент $x_{1}(t, w, \varepsilon), z_{1}(t, w, \varepsilon)$ решения задачи Коши (3.3), (3.4) при $\varepsilon \rightarrow 0$ имеют место асимптотические представления

$$
\begin{gathered}
x_{1}=\bar{x}\left(t, z_{0}, \varepsilon\right)+O\left(\varepsilon^{2}\right) e^{t}, \quad \frac{\partial x_{1}}{\partial y_{0}}=O\left(\frac{\varepsilon^{2}}{\ln (1 / \varepsilon)}\right)(t+1) e^{2 t}, \\
\frac{\partial x_{1}}{\partial z_{0}}=\frac{\partial \bar{x}}{\partial z_{0}}\left(t, z_{0}, \varepsilon\right)+O\left(\frac{\varepsilon^{2}}{\ln (1 / \varepsilon)}\right)(t+1) e^{2 t}, \\
z_{1}=\bar{z}\left(t, z_{0}, \varepsilon\right)+O\left(\varepsilon^{2}\right) e^{t}, \quad \frac{\partial z_{1}}{\partial y_{0}}=O\left(\varepsilon^{2}\right)(t+1) e^{t}, \\
\frac{\partial z_{1}}{\partial z_{0}}=\frac{\partial \bar{z}}{\partial z_{0}}\left(t, z_{0}, \varepsilon\right)+O\left(\varepsilon^{2}\right)(t+1) e^{t},
\end{gathered}
$$

əде

$$
\begin{aligned}
& \bar{x}\left(t, z_{0}, \varepsilon\right)=\exp \left\{t-\frac{z_{0}}{\ln (1 / \varepsilon)}\left(e^{t}-1\right)-\varepsilon\left(e^{t}-1-t\right)\right\}, \\
& \bar{z}\left(t, z_{0}, \varepsilon\right)=z_{0} e^{t}+\left(e^{t}-1\right) \varepsilon \ln \frac{1}{\varepsilon} .
\end{aligned}
$$

Что же касается компоненты $y_{1}(t, w, \varepsilon)$, то она на любом отрезке времени вида $\delta \leqslant t \leqslant \bar{t}, \delta=$ const $>0$, при $\varepsilon \rightarrow 0$ допускает представления

$$
\begin{gathered}
y_{1}=\bar{y}\left(t, z_{0}, \varepsilon\right)+O\left(\varepsilon^{2}\right) e^{-t}, \quad \frac{\partial y_{1}}{\partial y_{0}}=O\left(\frac{\varepsilon^{2}}{\ln (1 / \varepsilon)}\right)(t+1), \\
\frac{\partial y_{1}}{\partial z_{0}}=\frac{\partial \bar{y}}{\partial z_{0}}\left(t, z_{0}, \varepsilon\right)+O\left(\frac{\varepsilon^{2}}{\ln (1 / \varepsilon)}\right)(t+1),
\end{gathered}
$$

¿əe

$$
\bar{y}\left(t, z_{0}, \varepsilon\right)=\frac{1}{\bar{x}}+\frac{2 \varepsilon}{\bar{x}^{2}}-\frac{\bar{z}}{\bar{x}^{2}} \cdot \frac{\varepsilon}{\ln (1 / \varepsilon)},
$$

$a \bar{x}, \bar{z}-$ функиии (3.9), (3.10). Кроме того, остатки в формулах (3.5)-(3.8), (3.11), (3.12) имеют указанные порядки малости равномерно по $t, w$.

ДоказАтельство. Анализ задачи Коши (3.3), (3.4) проведем при априорных предположениях

$$
y_{1} \leqslant M_{1} e^{-t}, \quad\left|\frac{\partial y_{1}}{\partial y_{0}}\right| \leqslant M_{2}, \quad\left|\frac{\partial y_{1}}{\partial z_{0}}\right| \leqslant M_{3} .
$$

Здесь и ниже через $M_{1}, M_{2}$ и т. д. обозначаются некоторые универсальные (не зависящие от $t, w, \varepsilon)$ положительные константы, точные значения которых несущественны.

Обратимся сначала к третьему уравнению системы (3.3), считая в нем $y_{1}$ уже известной функцией, удовлетворяющей оценкам (3.14). Объединяя эти оценки с формулой

$$
z_{1}=z_{0} \exp \left\{\int_{0}^{t}\left(1-\varepsilon^{2} y_{1}\right) d \tau\right\}+\int_{0}^{t} \exp \left\{\int_{\tau}^{t}\left(1-\varepsilon^{2} y_{1}\right) d s\right\} d \tau \cdot \varepsilon \ln \frac{1}{\varepsilon},
$$


нетрудно убедиться в справедливости при $\varepsilon \rightarrow 0$ для компоненты $z_{1}(t, w, \varepsilon)$ равномерных по $t, w$ асимптотических представлений (3.7), (3.8).

В случае компоненты $x_{1}(t, w, \varepsilon)$ рассуждения аналогичны: обращаясь к первому уравнению системы (3.3) и считая в нем $z_{1}$ уже известной функцией, для $x_{1}$ получаем аналогичную (3.15) явную формулу. Далее, учитывая в этой формуле соотношения (3.7), (3.8), приходим к требуемым асимптотическим представлениям (3.5), (3.6).

Для отыскания асимптотики компоненты $y_{1}(t, w, \varepsilon)$ заметим следующее: из структуры правых частей системы (3.3) и из результатов работ [2], [10] вытекает существование у этой системы экспоненциально орбитально устойчивого (с показателем экспоненты порядка $\left.\varepsilon^{-1}\right)$ гладкого инвариантного многообразия медленных движений. Упомянутое многообразие задается равенствами вида

$$
\begin{gathered}
\left\{\left(x_{1}, y_{1}, z_{1}\right): y_{1}=H\left(x_{1}, z_{1}, \varepsilon\right),\left(x_{1}, z_{1}\right) \in \mathcal{V}_{1}\right\}, \\
\mathcal{V}_{1}=\left\{\left(x_{1}, z_{1}\right): 1 \leqslant x_{1} \leqslant M_{4} \ln ^{\lambda_{1}} \frac{1}{\varepsilon},\left|z_{1}\right| \leqslant M_{5} \ln ^{\lambda_{1}} \frac{1}{\varepsilon}\right\},
\end{gathered}
$$

где постоянные $M_{4}, M_{5}>0$ произвольно фиксированы, а функция $H$ при $\varepsilon \rightarrow 0$ допускает равномерные по $\left(x_{1}, z_{1}\right) \in \mathcal{V}_{1}$ асимптотические представления

$$
\begin{aligned}
H & =\frac{1}{x_{1}}\left(1+\frac{\varepsilon}{x_{1}}\left(2-\frac{z_{1}}{\ln (1 / \varepsilon)}\right)+\frac{1}{x_{1}^{2}} O\left(\varepsilon^{2}\right)\right), \\
\frac{\partial H}{\partial x_{1}} & =-\frac{1}{x_{1}^{2}}\left(1+\frac{2 \varepsilon}{x_{1}}\left(2-\frac{z_{1}}{\ln (1 / \varepsilon)}\right)+\frac{1}{x_{1}^{2}} O\left(\varepsilon^{2}\right)\right), \\
\frac{\partial H}{\partial z_{1}} & =-\frac{1}{x_{1}^{2}}\left(\frac{\varepsilon}{\ln (1 / \varepsilon)}+\frac{1}{x_{1}} O\left(\frac{\varepsilon^{2}}{\ln (1 / \varepsilon)}\right)\right) .
\end{aligned}
$$

Далее, при увеличении $t$ происходит асимптотически быстрое "падение" фазовой точки $\left(x_{1}(t, w, \varepsilon), y_{1}(t, w, \varepsilon), z_{1}(t, w, \varepsilon)\right)$ на многообразие (3.16) и последующее движение в асимптотически малой его окрестности (по прошествии времени $t=\delta$, $\delta=$ const $>0$, близость упомянутой фазовой точки к многообразию (3.16) оценивается сверху величиной порядка $e^{-c / \varepsilon}, c=$ const $\left.>0\right)$. Тем самым, отбрасывая экспоненциально малые добавки, при $t \geqslant \delta$ имеем

$$
y_{1}(t, w, \varepsilon)=\left.H\left(x_{1}, z_{1}, \varepsilon\right)\right|_{x_{1}=x_{1}(t, w, \varepsilon), z_{1}=z_{1}(t, w, \varepsilon)} .
$$

Для того чтобы обеспечить применимость соотношения (3.20) на интересующем нас асимптотически большом отрезке времени $\delta \leqslant t \leqslant \bar{t}$, подберем соответствующим образом постоянные $M_{4}, M_{5}$ из (3.16) (выбор этих постоянных означает, что мы рассматриваем не все многообразие медленных движений, а лишь определенный его участок). Опираясь на асимптотические представления для $x_{1}(t, w, \varepsilon), z_{1}(t, w, \varepsilon)$ (см. (3.5), (3.7)) и учитывая оценку $\lambda_{1}<1$, нетрудно увидеть, что требуемые в $(3.20)$ условия

$$
1 \leqslant x_{1}(t, w, \varepsilon) \leqslant M_{4} \ln ^{\lambda_{1}} \frac{1}{\varepsilon}, \quad z_{1}(t, w, \varepsilon) \leqslant M_{5} \ln ^{\lambda_{1}} \frac{1}{\varepsilon}
$$

будут заведомо выполняться при $M_{4}>1, M_{5}>\max _{w \in \Omega} z_{0}$. 
Итак, мы убедились, что при $\delta \leqslant t \leqslant \bar{t}$ оценки (3.21) действительно имеют место, а значит, при указанных $t$ справедлива и формула (3.20). Что же касается интересующих нас асимптотических равенств (3.11)-(3.13), то они получаются из нее при повторном разложении по $\varepsilon$ с учетом уже установленной информации (3.5)-(3.10), (3.17)-(3.19).

Отдельно остановимся на проверке априорных предположений (3.14), при которых были выведены представления (3.5)-(3.13). Отметим сразу, что на промежутке $0 \leqslant t \leqslant \delta$ конечной длины их справедливость очевидна. В случае же асимптотически большого промежутка $\delta \leqslant t \leqslant \bar{t}$ из формул (3.11)-(3.13) заключаем, что нужные оценки (3.14) автоматически выполняются при всех достаточно малых $\varepsilon>0$ с любыми фиксированными константами $M_{1}>1, M_{2}, M_{3}>0$. Лемма доказана.

На втором этапе выполним в системе (1.8) замену времени

$$
t=\ln \ln \frac{1}{\varepsilon}+\tau
$$

и аналогичные (3.2) замены переменных

$$
x=x_{2} \ln \frac{1}{\varepsilon}, \quad y=\frac{\varepsilon^{3}}{\ln (1 / \varepsilon)} y_{2}, \quad z=\varepsilon z_{2} .
$$

В результате приходим к системе

$$
\frac{d x_{2}}{d \tau}=\left(1-z_{2}\right) x_{2}+\varepsilon \mu, \quad \mu \frac{d y_{2}}{d \tau}=\left(\mu-x_{2}\right) y_{2}+1, \quad \frac{d z_{2}}{d \tau}=\left(1-\varepsilon \mu y_{2}\right) z_{2}+\varepsilon,
$$

где $\mu=\varepsilon / \ln (1 / \varepsilon)$. Полученную систему будем рассматривать на отрезке $\tau_{1} \leqslant \tau \leqslant \tau_{2}$, где

$$
\tau_{1}=-\left(1-\lambda_{1}\right) \ln \ln \frac{1}{\varepsilon},
$$

a $\tau_{2}=\tau_{2}\left(z_{0}, \varepsilon\right)$ - положительный корень уравнения

$$
\exp \left(\tau-z_{0} e^{\tau}\right)=\varepsilon \ln ^{n_{1}} \frac{1}{\varepsilon}
$$

при некотором фиксированном $n_{1} \in \mathbb{N}$. Несложный анализ уравнения (3.26) приводит к выводу, что при $\varepsilon \rightarrow 0$

$$
\begin{gathered}
\tau_{2}=\ln \left(\frac{1}{z_{0}} \ln \frac{1}{\varepsilon}\right)+\left(1-n_{1}\right) \frac{\ln \ln (1 / \varepsilon)}{\ln (1 / \varepsilon)}-\frac{\ln z_{0}}{\ln (1 / \varepsilon)}+O\left(\frac{\ln ^{2}(\ln (1 / \varepsilon))}{\ln ^{2}(1 / \varepsilon)}\right), \\
\frac{\partial \tau_{2}}{\partial z_{0}}=\frac{e^{\tau_{2}}}{1-z_{0} e^{\tau_{2}}}=-\frac{1}{z_{0}}+O\left(\frac{1}{\ln (1 / \varepsilon)}\right) .
\end{gathered}
$$

В соответствии с предыдущим этапом и заменами (3.2), (3.22), (3.23) мы должны дополнить систему (3.24) начальными условиями

$$
\left.x_{2}\right|_{\tau=\tau_{1}}=x_{2,0}(w, \varepsilon),\left.\quad y_{2}\right|_{\tau=\tau_{1}}=y_{2,0}(w, \varepsilon),\left.\quad z_{2}\right|_{\tau=\tau_{1}}=z_{2,0}(w, \varepsilon),
$$

где

$$
x_{2,0}=\frac{x_{1}(\bar{t}, w, \varepsilon)}{\ln (1 / \varepsilon)}, \quad y_{2,0}=y_{1}(\bar{t}, w, \varepsilon) \ln \frac{1}{\varepsilon}, \quad z_{2,0}=\frac{z_{1}(\bar{t}, w, \varepsilon)}{\ln (1 / \varepsilon)}
$$


а $\tau_{1}$ - момент времени (3.25). Обозначим через $x_{2}(\tau, w, \varepsilon), y_{2}(\tau, w, \varepsilon), z_{2}(\tau, w, \varepsilon)$ компоненты решения задачи Коши (3.24), (3.28) и выявим их асимптотику при $\varepsilon \rightarrow 0$.

Подчеркнем, что асимптотика при $\varepsilon \rightarrow 0$ фигурирующих в (3.28) функций $x_{2,0}$, $y_{2,0}, z_{2,0}$ уже известна: из соотношений (3.5)-(3.13) вытекают равномерные по $w \in \Omega$ представления

$$
\begin{gathered}
x_{2,0}=\exp \left(-\frac{z_{0}}{\ln ^{1-\lambda_{1}}(1 / \varepsilon)}+\frac{z_{0}}{\ln (1 / \varepsilon)}\right) \ln ^{\lambda_{1}-1} \frac{1}{\varepsilon}+O\left(\varepsilon \ln ^{2 \lambda_{1}-1} \frac{1}{\varepsilon}\right), \\
\frac{\partial x_{2,0}}{\partial y_{0}}=O\left(\frac{\varepsilon^{2} \ln \ln (1 / \varepsilon)}{\ln ^{2-2 \lambda_{1}}(1 / \varepsilon)}\right), \\
\frac{\partial x_{2,0}}{\partial z_{0}}=\frac{\partial}{\partial z_{0}}\left[\exp \left(-\frac{z_{0}}{\ln ^{1-\lambda_{1}}(1 / \varepsilon)}+\frac{z_{0}}{\ln (1 / \varepsilon)}\right) \ln ^{\lambda_{1}-1} \frac{1}{\varepsilon}\right]+O\left(\varepsilon \ln ^{3 \lambda_{1}-2} \frac{1}{\varepsilon}\right) ; \\
\left.\frac{\partial y_{2,0}}{\partial y_{0}}=O\left(\varepsilon^{2} \ln \ln \frac{1}{\varepsilon}\right), \frac{z_{0}}{\ln (1 / \varepsilon)}\right) \ln ^{1-\lambda_{1}} \frac{1}{\varepsilon}+O\left(\varepsilon \ln \frac{1}{\varepsilon}\right), \\
\frac{\partial y_{2,0}}{\partial z_{0}}=\frac{\partial}{\partial z_{0}}\left[\exp \left(\frac{z_{0}}{\ln ^{1-\lambda_{1}}(1 / \varepsilon)}-\frac{z_{0}}{\ln (1 / \varepsilon)}\right) \ln ^{1-\lambda_{1}} \frac{1}{\varepsilon}\right]+O\left(\varepsilon \ln \frac{1}{\varepsilon}\right) \\
z_{2,0}=z_{0} \ln ^{\lambda_{1}-1} \frac{1}{\varepsilon}+O\left(\varepsilon \ln \lambda^{\lambda_{1}} \frac{1}{\varepsilon}\right), \frac{\partial z_{2,0}}{\partial y_{0}}=O\left(\frac{\varepsilon^{2} \ln \ln (1 / \varepsilon)}{\ln ^{1-\lambda_{1}}(1 / \varepsilon)}\right) \\
\frac{\partial z_{2,0}}{\partial z_{0}}=\ln ^{\lambda_{1}-1} \frac{1}{\varepsilon}+O\left(\frac{\varepsilon^{2} \ln \ln (1 / \varepsilon)}{\ln ^{1-\lambda_{1}}(1 / \varepsilon)}\right) \cdot
\end{gathered}
$$

Что касается самого решения задачи (3.24), (3.28), то для него справедлива следующая

ЛЕмма 2. На отрезке $\tau_{1} \leqslant \tau \leqslant \tau_{2}$ при $\varepsilon \rightarrow 0$ компоненты $x_{2}(\tau, w, \varepsilon), z_{2}(\tau, w, \varepsilon)$ допускают равномерные по $\tau, w$ асимптотические представления

$$
\begin{aligned}
x_{2} & =\left(1+O\left(\varepsilon \ln \frac{1}{\varepsilon}\right) e^{\tau}\right) \exp \left\{\tau-z_{0} e^{\tau}+\frac{z_{0}}{\ln (1 / \varepsilon)}\right\} \\
\frac{\partial x_{2}}{\partial y_{0}} & =O\left(\varepsilon^{2} \ln \ln \frac{1}{\varepsilon}\right) \exp \left(2 \tau-z_{0} e^{\tau}\right) \\
\frac{\partial x_{2}}{\partial z_{0}} & =\left(1+O\left(\varepsilon \ln \frac{1}{\varepsilon}\right) e^{\tau}\right) \frac{\partial}{\partial z_{0}} \exp \left\{\tau-z_{0} e^{\tau}+\frac{z_{0}}{\ln (1 / \varepsilon)}\right\} ; \\
z_{2} & =\left(z_{0}+O\left(\varepsilon \ln \frac{1}{\varepsilon}\right)\right) e^{\tau}, \quad \frac{\partial z_{2}}{\partial y_{0}}=O\left(\varepsilon^{2} \ln \ln \frac{1}{\varepsilon}\right) e^{\tau} \\
\frac{\partial z_{2}}{\partial z_{0}} & =\left(1+O\left(\frac{\varepsilon}{\ln ^{n_{1}-1}(1 / \varepsilon)}\right)\right) e^{\tau}
\end{aligned}
$$


а для компоненты $y_{2}(\tau, w, \varepsilon)$ на любом отрезке вида $\tau_{1}+\delta \leqslant \tau \leqslant \tau_{2}$, где постоянная $\delta>0$ произвольно фиксирована, имеют место равномерные по $\tau, w$ асимптотические представления

$$
\begin{aligned}
y_{2}= & \left(1+O\left(\varepsilon \ln \frac{1}{\varepsilon}\right) e^{\tau}+O\left(\frac{\varepsilon}{\ln (1 / \varepsilon)}\right)\left(e^{\tau}+1\right) \exp \left(-\tau+z_{0} e^{\tau}\right)\right) \times \\
& \times \exp \left\{-\tau+z_{0} e^{\tau}-\frac{z_{0}}{\ln (1 / \varepsilon)}\right\}, \\
\frac{\partial y_{2}}{\partial y_{0}}= & O\left(\varepsilon^{2} \ln \ln \frac{1}{\varepsilon}\right) \exp \left(z_{0} e^{\tau}\right), \\
\frac{\partial y_{2}}{\partial z_{0}}= & \left(1+O\left(\varepsilon \ln \frac{1}{\varepsilon}\right) e^{\tau}+O\left(\frac{\varepsilon}{\ln (1 / \varepsilon)}\right)\left(e^{\tau}+1\right) \exp \left(-\tau+z_{0} e^{\tau}\right)\right) \times \\
& \times \frac{\partial}{\partial z_{0}} \exp \left\{-\tau+z_{0} e^{\tau}-\frac{z_{0}}{\ln (1 / \varepsilon)}\right\} .
\end{aligned}
$$

ДокАзАтельство. Обоснование леммы базируется на тех же идеях, что и доказательство леммы 1. А именно, будем считать, что при некоторых $M_{1}, M_{2}, M_{3}>0$ выполнены априорные оценки

$$
y_{2} \leqslant \frac{M_{1}}{\varepsilon \ln ^{n_{1}}(1 / \varepsilon)}, \quad\left|\frac{\partial y_{2}}{\partial y_{0}}\right| \leqslant M_{2}, \quad\left|\frac{\partial y_{2}}{\partial z_{0}}\right| \leqslant \frac{M_{3}}{\varepsilon \ln ^{n_{1}-1}(1 / \varepsilon)} .
$$

Тогда, предполагая функцию $y_{2}$ уже известной и дополняя третье уравнение системы (3.24) начальным условием (3.31) при $\tau=\tau_{1}$, для $z_{2}$ после некоторых преобразований получаем равномерные по $\tau \in\left[\tau_{1}, \tau_{2}\right], w \in \Omega$ асимптотические формулы (3.33). В свою очередь, опираясь на асимптотические представления (3.29), (3.33) и считая известной функцию $z_{2}$, из первого уравнения системы (3.24) выводим группу формул (3.32).

Как и в предыдущем случае, при анализе компоненты $y_{2}(\tau, w, \varepsilon)$ воспользуемся результатами из [2], [10]. Из упомянутых результатов вытекает существование у системы (3.24) аналогичного (3.16) инвариантного многообразия медленных движений:

$$
\begin{gathered}
\left\{\left(x_{2}, y_{2}, z_{2}\right): y_{2}=H\left(x_{2}, z_{2}, \varepsilon, \mu\right),\left(x_{2}, z_{2}\right) \in \mathcal{V}_{2}\right\}, \\
\mathcal{V}_{2}=\left\{\left(x_{2}, z_{2}\right): M_{4} \varepsilon \ln ^{n_{1}} \frac{1}{\varepsilon} \leqslant x_{2} \leqslant M_{5},\left|z_{2}\right| \leqslant M_{6} \ln \frac{1}{\varepsilon}\right\},
\end{gathered}
$$

где $M_{4}, M_{5}, M_{6}>0$ - произвольно фиксированные константы, а для $H$ при $\mu \rightarrow 0$ справедливы равномерные по $\left(x_{2}, z_{2}\right) \in \mathcal{V}_{2}$ и $\varepsilon$ асимптотические равенства

$$
\begin{aligned}
H & =\frac{1}{x_{2}}\left(1+\mu \frac{2-z_{2}}{x_{2}}+\frac{1}{x_{2}^{2}} O\left(\mu^{2}\right)\right), \\
\frac{\partial H}{\partial x_{2}} & =-\frac{1}{x_{2}^{2}}\left(1+2 \mu \frac{2-z_{2}}{x_{2}}+\frac{1}{x_{2}^{2}} O\left(\mu^{2}\right)\right), \\
\frac{\partial H}{\partial z_{2}} & =\frac{1}{x_{2}}\left(-\frac{\mu}{x_{2}}+\frac{1}{x_{2}^{2}} O\left(\mu^{2}\right)\right) .
\end{aligned}
$$

При увеличении $\tau$ от $\tau=\tau_{1}$ до $\tau=\tau_{1}+\delta$, где $\delta=$ const $>0$, интересующая нас траектория $\left(x_{2}(\tau, w, \varepsilon), y_{2}(\tau, w, \varepsilon), z_{2}(\tau, w, \varepsilon)\right)$ системы (3.24) успевает приблизиться 
к многообразию (3.36) на расстояние порядка $\exp \left(-c \ln ^{\lambda_{1}}(1 / \varepsilon) / \varepsilon\right), c=$ const $>0$. А это значит, что при $\tau \geqslant \tau_{1}+\delta$ функция $y_{2}(\tau, w, \varepsilon)$ с точностью до экспоненциально малой добавки задается аналогичным (3.20) равенством

$$
y_{2}(\tau, w, \varepsilon)=\left.H\left(x_{2}, z_{2}, \varepsilon, \mu\right)\right|_{x_{2}=x_{2}(\tau, w, \varepsilon), z_{2}=z_{2}(\tau, w, \varepsilon), \mu=\varepsilon / \ln (1 / \varepsilon)} .
$$

Как и ранее, для того чтобы придать соотношению (3.38) законную силу, необходимо убедиться, что $\left(x_{2}(\tau, w, \varepsilon), z_{2}(\tau, w, \varepsilon)\right) \in \mathcal{V}_{2}$ при всех $\tau_{1}+\delta \leqslant \tau \leqslant \tau_{2}$. Из формул (3.32), (3.33) несложно вывести, что указанное включение заведомо справедливо при

$$
M_{4} \in(0,1), \quad M_{5}>e^{-1} \max _{w \in \Omega} \frac{1}{z_{0}}, \quad M_{6}>1 .
$$

Далее, считая неравенства (3.39) выполненными (напомним, что постоянные $M_{4}$, $M_{5}, M_{6}$ в нашем распоряжении), снова разложим правую часть (3.38) по $\varepsilon$. В результате, опираясь на представления (3.32), (3.33), (3.37), для $y_{2}$ получаем требуемую асимптотику (3.34).

Для завершения доказательства леммы осталось проверить априорные неравенства (3.35). Обратим внимание, что на отрезке $\tau_{1} \leqslant \tau \leqslant \tau_{1}+\delta$ конечной длины их справедливость очевидна, а на асимптотически большом отрезке $\tau_{1}+\delta \leqslant \tau \leqslant \tau_{2}$ эти условия вытекают из представлений (3.34). Точнее говоря, они выполняются при всех достаточно малых $\varepsilon>0$ с константами $M_{1}>1, M_{2}>0, M_{3}>\max _{w \in \Omega}\left(1 / z_{0}\right)$. Лемма доказана.

Прежде чем перейти к следующему шагу, введем в рассмотрение положительные корни $\tau=\bar{\tau}\left(z_{0}, \varepsilon\right)$ и $\tau=\tau_{3}\left(z_{0}, \varepsilon\right)$ уравнений $\exp \left(\tau-z_{0} e^{\tau}\right)=\varepsilon$ и $\exp \left(\tau-z_{0} e^{\tau}\right)=$ $\varepsilon / \ln ^{n_{2}}(1 / \varepsilon), n_{2} \in \mathbb{N}, n_{2} \geqslant 5$, соответственно. Нетрудно увидеть, что при $\varepsilon \rightarrow 0$ указанные корни допускают асимптотику

$$
\begin{gathered}
\bar{\tau}=\ln \left(\frac{1}{z_{0}} \ln \frac{1}{\varepsilon}\right)+\frac{\ln \ln (1 / \varepsilon)}{\ln (1 / \varepsilon)}-\frac{\ln z_{0}}{\ln (1 / \varepsilon)}+O\left(\frac{\ln ^{2}(\ln (1 / \varepsilon))}{\ln ^{2}(1 / \varepsilon)}\right), \\
\frac{\partial \bar{\tau}}{\partial z_{0}}=\frac{e^{\bar{\tau}}}{1-z_{0} e^{\bar{\tau}}}=-\frac{1}{z_{0}}+O\left(\frac{1}{\ln (1 / \varepsilon)}\right) ; \\
\tau_{3}=\ln \left(\frac{1}{z_{0}} \ln \frac{1}{\varepsilon}\right)+\left(1+n_{2}\right) \frac{\ln \ln (1 / \varepsilon)}{\ln (1 / \varepsilon)}-\frac{\ln z_{0}}{\ln (1 / \varepsilon)}+O\left(\frac{\ln ^{2}(\ln (1 / \varepsilon))}{\ln ^{2}(1 / \varepsilon)}\right), \\
\frac{\partial \tau_{3}}{\partial z_{0}}=\frac{e^{\tau_{3}}}{1-z_{0} e^{\tau_{3}}}=-\frac{1}{z_{0}}+O\left(\frac{1}{\ln (1 / \varepsilon)}\right) .
\end{gathered}
$$

На третьем этапе рассмотрим отрезок $\tau_{2} \leqslant \tau \leqslant \tau_{3}$. В связи с этим выполним в исходной системе (1.8) замену времени (3.22) и положим

$$
x=x_{3} \varepsilon \ln \frac{1}{\varepsilon}, \quad y=\frac{\varepsilon^{2}}{\ln (1 / \varepsilon)} y_{3}, \quad z=z_{3} \varepsilon \ln \frac{1}{\varepsilon}, \quad \tau=\bar{\tau}+\frac{s}{\ln (1 / \varepsilon)} .
$$

Здесь новое время $s$ меняется на отрезке $s_{1} \leqslant s \leqslant s_{2}, s_{j}=\left(\tau_{j+1}-\bar{\tau}\right) \ln (1 / \varepsilon), j=1,2$, концы которого согласно $(3.27),(3.40),(3.41)$ допускают при $\varepsilon \rightarrow 0$ асимптотику

$$
\begin{array}{ll}
s_{1}=-n_{1} \ln \ln \frac{1}{\varepsilon}+O\left(\frac{\ln ^{2}(\ln (1 / \varepsilon))}{\ln (1 / \varepsilon)}\right), & \frac{\partial s_{1}}{\partial z_{0}}=O\left(\frac{\ln (\ln (1 / \varepsilon))}{\ln (1 / \varepsilon)}\right), \\
s_{2}=n_{2} \ln \ln \frac{1}{\varepsilon}+O\left(\frac{\ln ^{2}(\ln (1 / \varepsilon))}{\ln (1 / \varepsilon)}\right), & \frac{\partial s_{2}}{\partial z_{0}}=O\left(\frac{\ln (\ln (1 / \varepsilon))}{\ln (1 / \varepsilon)}\right) .
\end{array}
$$


После замен (3.42) система (1.8) принимает вид

$$
\begin{aligned}
\frac{d x_{3}}{d s} & =\left(\frac{1}{\ln (1 / \varepsilon)}-z_{3}\right) x_{3}+\frac{\varepsilon}{\ln ^{2}(1 / \varepsilon)} \\
\frac{d y_{3}}{d s} & =\left(\frac{1}{\ln (1 / \varepsilon)}-x_{3}\right) y_{3}+1 \\
\frac{d z_{3}}{d s} & =\left(\frac{1}{\ln (1 / \varepsilon)}-\frac{\varepsilon}{\ln ^{2}(1 / \varepsilon)} y_{3}\right) z_{3}+\frac{\varepsilon}{\ln ^{2}(1 / \varepsilon)} .
\end{aligned}
$$

Согласно предыдущему шагу получившуюся систему следует дополнить начальными условиями

$$
\left.x_{3}\right|_{s=s_{1}}=x_{3,0}(w, \varepsilon),\left.\quad y_{3}\right|_{s=s_{1}}=y_{3,0}(w, \varepsilon),\left.\quad z_{3}\right|_{s=s_{1}}=z_{3,0}(w, \varepsilon),
$$

где

$$
x_{3,0}=\frac{x_{2}\left(\tau_{2}, w, \varepsilon\right)}{\varepsilon}, \quad y_{3,0}=\varepsilon y_{2}\left(\tau_{2}, w, \varepsilon\right), \quad z_{3,0}=\frac{z_{2}\left(\tau_{2}, w, \varepsilon\right)}{\ln (1 / \varepsilon)} .
$$

Прежде всего выясним асимптотику функций (3.46). Для этого нам понадобятся формулы (3.27), (3.32)-(3.34). Опираясь на них, нетрудно убедиться, что при $\varepsilon \rightarrow 0$ равномерно по $w \in \Omega$ имеют место асимптотические представления

$$
\begin{gathered}
x_{3,0}=\left(1+O\left(\frac{1}{\ln (1 / \varepsilon)}\right)\right) \ln ^{n_{1}} \frac{1}{\varepsilon}, \quad \frac{\partial x_{3,0}}{\partial y_{0}}=O\left(\varepsilon^{2} \ln ^{n_{1}+1} \frac{1}{\varepsilon} \cdot \ln \ln \frac{1}{\varepsilon}\right), \\
\frac{\partial x_{3,0}}{\partial z_{0}}=O\left(\ln ^{n_{1}-1} \frac{1}{\varepsilon}\right) ; \\
y_{3,0}=\left(1+O\left(\frac{1}{\ln (1 / \varepsilon)}\right)\right) \frac{1}{\ln ^{n_{1}}(1 / \varepsilon)}, \quad \frac{\partial y_{3,0}}{\partial y_{0}}=O\left(\frac{\varepsilon^{2} \ln \ln (1 / \varepsilon)}{\ln ^{n_{1}-1}(1 / \varepsilon)}\right), \\
z_{3,0}=1+O\left(\frac{\ln \ln (1 / \varepsilon)}{\ln (1 / \varepsilon)}\right), \quad \frac{\partial z_{3,0}}{\partial z_{0}}=O\left(\varepsilon^{2} \ln \ln \frac{1}{\varepsilon}\right), \\
\frac{\partial z_{3,0}}{\partial z_{0}}=O\left(\frac{1}{\ln (1 / \varepsilon)}\right) .
\end{gathered}
$$

Обозначим компоненты решения задачи Коши $(3.44),(3.45)$ через $x_{3}(s, w, \varepsilon)$, $y_{3}(s, w, \varepsilon), z_{3}(s, w, \varepsilon)$. Справедливо следующее утверждение.

ЛЕмма 3. На отрезке $s_{1} \leqslant s \leqslant s_{2}$ при $\varepsilon \rightarrow 0$ выполняются равномерные по $s, w$ асимптотические равенства

$$
\begin{gathered}
x_{3}=\left(1+O\left(\frac{\ln ^{2} \ln (1 / \varepsilon)}{\ln (1 / \varepsilon)}\right)\right) e^{-s}, \quad \frac{\partial x_{3}}{\partial y_{0}}=O\left(\frac{\varepsilon \ln \ln (1 / \varepsilon)}{\ln ^{2}(1 / \varepsilon)}\right) e^{-s}, \\
\frac{\partial x_{3}}{\partial z_{0}}=O\left(\frac{\ln \ln (1 / \varepsilon)}{\ln (1 / \varepsilon)}\right) e^{-s}
\end{gathered}
$$




$$
\begin{gathered}
y_{3}=y_{3}^{0}(s)+O\left(\frac{\ln ^{2} \ln (1 / \varepsilon)}{\ln (1 / \varepsilon)}\right), \quad \frac{\partial y_{3}}{\partial y_{0}}=O\left(\frac{\varepsilon \ln \ln (1 / \varepsilon)}{\ln ^{2}(1 / \varepsilon)}\right), \\
\frac{\partial y_{3}}{\partial z_{0}}=O\left(\frac{\ln \ln (1 / \varepsilon)}{\ln (1 / \varepsilon)}\right) ; \\
z_{3}=1+O\left(\frac{\ln \ln (1 / \varepsilon)}{\ln (1 / \varepsilon)}\right), \quad \frac{\partial z_{3}}{\partial y_{0}}=O\left(\frac{\varepsilon}{\ln ^{2}(1 / \varepsilon)}\right), \quad \frac{\partial z_{3}}{\partial z_{0}}=O\left(\frac{1}{\ln (1 / \varepsilon)}\right),
\end{gathered}
$$

где

$$
y_{3}^{0}(s)=\int_{-\infty}^{s} \exp \left\{e^{-s}-e^{-\sigma}\right\} d \sigma .
$$

ДокАзАТЕЛЬство. Обоснование леммы проведем при априорных предположениях

$$
y_{3} \leqslant M_{1} \ln \ln \frac{1}{\varepsilon}, \quad\left|\frac{\partial y_{3}}{\partial y_{0}}\right| \leqslant M_{2}, \quad\left|\frac{\partial y_{3}}{\partial z_{0}}\right| \leqslant M_{3} .
$$

Как обычно, будем считать, что в третьем уравнении системы (3.44) функция $y_{3}$ известна и обладает свойствами (3.54). Объединяя эти свойства с асимптотическими представлениями (3.43), (3.49) и анализируя получившуюся задачу Коши для $z_{3}$, приходим к группе формул (3.52). Аналогичным образом рассуждаем и в случае $x_{3}$ : рассматриваем первое уравнение системы (3.44), предполагая в нем функцию $z_{3}$ уже известной и дополняя его при $s=s_{1}$ начальным условием (3.47). Опираясь затем на асимптотические представления (3.43), (3.47), (3.52), получаем очередную группу формул (3.50).

В случае компоненты $y_{3}$ действуем по той же схеме, т. е. анализируем второе уравнение системы (3.44) с начальным условием (3.48) при $s=s_{1}$ в предположении, что известной является функция $x_{3}$. При исследовании этой задачи Коши помимо асимптотических равенств (3.43), (3.48), (3.50) используются асимптотические свойства функции (3.53):

$$
\begin{gathered}
y_{3}^{0}(s)=s+c_{0}+O\left(s e^{-s}\right), \quad s \rightarrow+\infty, \\
c_{0}=\int_{-\infty}^{0} \exp \left(-e^{-\sigma}\right) d \sigma+\int_{0}^{+\infty}\left[\exp \left(-e^{-\sigma}\right)-1\right] d \sigma ; \\
y_{3}^{0}(s)=e^{s}+O\left(e^{2 s}\right), \quad s \rightarrow-\infty .
\end{gathered}
$$

Объединяя перечисленные факты и проводя соответствующий подсчет, для $y_{3}$ приходим к требуемым формулам (3.51).

Для придания проделанным выше построениям строгого смысла осталось убедиться в справедливости условий (3.54). Из формул (3.43), (3.51), (3.55), (3.56) вытекает, что эти условия заведомо выполняются с константами $M_{1}>n_{2}, M_{2}, M_{3}>0$. Лемма доказана.

На четвертом этапе сделаем в системе (1.8) замену времени (3.22) и положим в ней

$$
x=\varepsilon x_{4}, \quad y=\varepsilon^{2} y_{4}, \quad z=z_{4} \varepsilon \ln \frac{1}{\varepsilon}, \quad \theta=\tau-\tau_{3} .
$$

В результате приходим к системе

$$
\nu \frac{d x_{4}}{d \theta}=\left(\nu-z_{4}\right) x_{4}+\varepsilon \nu, \quad \frac{d y_{4}}{d \theta}=\left(1-x_{4}\right) y_{4}+1, \quad \frac{d z_{4}}{d \theta}=\left(1-\varepsilon y_{4}\right) z_{4}+\varepsilon \nu,
$$


где $\nu=1 / \ln (1 / \varepsilon)$. Всюду ниже считаем, что новое время $\theta$ из (3.57) меняется на отрезке $0 \leqslant \theta \leqslant \bar{\theta}$, где $\bar{\theta}=\lambda_{2} \ln (1 / \varepsilon)-\tau_{3}, \lambda_{2}=$ const $\in(0,1)$. Саму же систему $(3.58)$ в соответствии с предыдущим шагом дополним начальными условиями

$$
\left.x_{4}\right|_{\theta=0}=x_{4,0}(w, \varepsilon),\left.\quad y_{4}\right|_{\theta=0}=y_{4,0}(w, \varepsilon),\left.\quad z_{4}\right|_{\theta=0}=z_{4,0}(w, \varepsilon),
$$

где

$$
x_{4,0}=x_{3}\left(s_{2}, w, \varepsilon\right) \ln \frac{1}{\varepsilon}, \quad y_{4,0}=\frac{y_{3}\left(s_{2}, w, \varepsilon\right)}{\ln (1 / \varepsilon)}, \quad z_{4,0}=z_{3}\left(s_{2}, w, \varepsilon\right) .
$$

При анализе задачи Коши (3.58), (3.59) сначала разберемся с асимптотикой начальных условий (3.60). Из уже установленных представлений (3.43), (3.50)-(3.53) вытекает справедливость при $\varepsilon \rightarrow 0$ равномерных по $w \in \Omega$ асимптотических формул

$$
\begin{gathered}
x_{4,0}=\frac{1}{\ln ^{n_{2}-1}(1 / \varepsilon)}\left(1+O\left(\frac{\ln ^{2} \ln (1 / \varepsilon)}{\ln (1 / \varepsilon)}\right)\right), \quad \frac{\partial x_{4,0}}{\partial y_{0}}=O\left(\frac{\varepsilon \ln \ln (1 / \varepsilon)}{\ln ^{n_{2}+1}(1 / \varepsilon)}\right), \\
\frac{\partial x_{4,0}}{\partial z_{0}}=O\left(\frac{\ln \ln (1 / \varepsilon)}{\left.\ln ^{n_{2}(1 / \varepsilon)}\right) ;}\right. \\
y_{4,0}=n_{2} \frac{\ln \ln (1 / \varepsilon)}{\ln (1 / \varepsilon)}+O\left(\frac{1}{\ln (1 / \varepsilon)}\right), \quad \frac{\partial y_{4,0}}{\partial y_{0}}=O\left(\frac{\varepsilon \ln \ln (1 / \varepsilon)}{\ln ^{3}(1 / \varepsilon)}\right), \\
\quad \frac{\partial y_{4,0}}{\partial z_{0}}=O\left(\frac{\ln \ln (1 / \varepsilon)}{\ln ^{2}(1 / \varepsilon)}\right) ; \\
z_{4,0}=1+O\left(\frac{\ln \ln (1 / \varepsilon)}{\ln (1 / \varepsilon)}\right), \quad \frac{\partial z_{4,0}}{\partial y_{0}}=O\left(\frac{\varepsilon}{\ln ^{2}(1 / \varepsilon)}\right), \quad \frac{\partial z_{4,0}}{\partial z_{0}}=O\left(\frac{1}{\ln (1 / \varepsilon)}\right) .
\end{gathered}
$$

Далее, обозначим через $x_{4}(\theta, w, \varepsilon), y_{4}(\theta, w, \varepsilon), z_{4}(\theta, w, \varepsilon)$ компоненты решения задачи (3.58), (3.59). Имеет место следующая

ЛЕмма 4. При любом фиксированном $\delta>\ln 3$ бункиии $x_{4}(\theta, w, \varepsilon), y_{4}(\theta, w, \varepsilon)$, $z_{4}(\theta, w, \varepsilon)$ допускают при $\varepsilon \rightarrow 0$ равномерные по $\theta, w$ асимптотические представления

$$
\begin{aligned}
x_{4}= \begin{cases}O\left(\frac{1}{\ln ^{n_{2}-1}(1 / \varepsilon)}\right) & n p u \quad 0 \leqslant \theta \leqslant \delta, \\
\left(1+O\left(\frac{\ln \ln (1 / \varepsilon)}{\ln (1 / \varepsilon)}\right)\right) \frac{\varepsilon}{\ln (1 / \varepsilon)} e^{-\theta} & n p u \quad \delta \leqslant \theta \leqslant \bar{\theta},\end{cases} \\
\frac{\partial x_{4}}{\partial y_{0}}=\left\{\begin{array}{lll}
O\left(\frac{\varepsilon}{\ln ^{n_{2}-2}(1 / \varepsilon)}\right) & n p u & 0 \leqslant \theta \leqslant \delta, \\
O\left(\varepsilon^{2}\right) e^{-\theta} & n p u & \delta \leqslant \theta \leqslant \bar{\theta},
\end{array}\right. \\
\frac{\partial x_{4}}{\partial z_{0}}=\left\{\begin{array}{lll}
O\left(\frac{\ln ^{n} \ln (1 / \varepsilon)}{\ln ^{n_{2}}(1 / \varepsilon)}\right) & \text { npu } & 0 \leqslant \theta \leqslant \delta, \\
O\left(\frac{\varepsilon}{\ln ^{2}(1 / \varepsilon)}\right) e^{-\theta} & n p u & \delta \leqslant \theta \leqslant \bar{\theta} ;
\end{array}\right.
\end{aligned}
$$




$$
\begin{gathered}
y_{4}=e^{\theta}-1+O\left(\frac{\ln \ln (1 / \varepsilon)}{\ln (1 / \varepsilon)}\right) e^{\theta}, \quad \frac{\partial y_{4}}{\partial y_{0}}=O\left(\frac{\varepsilon \ln \ln (1 / \varepsilon)}{\ln ^{3}(1 / \varepsilon)}\right) e^{\theta}, \\
\frac{\partial y_{4}}{\partial z_{0}}=O\left(\frac{\ln \ln (1 / \varepsilon)}{\ln ^{2}(1 / \varepsilon)}\right) e^{\theta} \\
z_{4}=\left(1+O\left(\frac{\ln \ln (1 / \varepsilon)}{\ln (1 / \varepsilon)}\right)\right) e^{\theta}, \quad \frac{\partial z_{4}}{\partial y_{0}}=O\left(\varepsilon \ln \frac{1}{\varepsilon}\right) e^{\theta}, \\
\frac{\partial z_{4}}{\partial z_{0}}=O\left(\frac{1}{\ln (1 / \varepsilon)}\right) e^{\theta} .
\end{gathered}
$$

ДокАзАтЕльство. Обратимся сначала к третьему уравнению системы (3.58), считая, что функция $y_{4}$ в нем известна и удовлетворяет априорным оценкам

$$
y_{4} \leqslant M_{1} \frac{\varepsilon^{-\lambda_{2}}}{\ln (1 / \varepsilon)}, \quad\left|\frac{\partial y_{4}}{\partial y_{0}}\right| \leqslant M_{2}, \quad\left|\frac{\partial y_{4}}{\partial z_{0}}\right| \leqslant M_{3} \varepsilon^{-\lambda_{2}} .
$$

Принимая во внимание приведенную информацию об $y_{4}$ и опираясь на формулы (3.63), нетрудно убедиться, что для $z_{4}$ получаются асимптотические представления (3.66).

Следующий шаг связан с рассмотрением первого уравнения системы (3.58) в предположении, что функция $z_{4}$ известна и обладает свойствами (3.66). Это уравнение является сингулярно возмущенным, и к нему применимы результаты [2], [10], из которых следует, что у этого уравнения имеется так называемое “медленное" решение $x_{*}(\theta, w, \varepsilon)$, допускающее при $\varepsilon \rightarrow 0$ равномерную по $\theta \in[0, \bar{\theta}], w \in \Omega$ асимптотику

$$
\begin{gathered}
x_{*}=\left(1+O\left(\frac{\ln \ln (1 / \varepsilon)}{\ln (1 / \varepsilon)}\right)\right) \frac{\varepsilon}{\ln (1 / \varepsilon)} e^{-\theta}, \quad \frac{\partial x_{*}}{\partial y_{0}}=O\left(\varepsilon^{2}\right) e^{-\theta}, \\
\frac{\partial x_{*}}{\partial z_{0}}=O\left(\frac{\varepsilon}{\ln ^{2}(1 / \varepsilon)}\right) e^{-\theta} .
\end{gathered}
$$

Что касается интересующего нас решения $x_{4}(\theta, w, \varepsilon)$ указанного уравнения, то в силу формул (3.61) оно отличается от (3.68) на величину

$$
O\left(\frac{1}{\ln ^{n_{2}-1}(1 / \varepsilon)}\right) \exp \left(-\left(e^{\theta}-1\right)\left(1+O\left(\frac{\ln \ln (1 / \varepsilon)}{\ln (1 / \varepsilon)}\right)\right) \ln \frac{1}{\varepsilon}\right) .
$$

В частности, при $\theta \in[\delta, \bar{\theta}]$, где $\delta=$ const $>\ln 3$, остаток (3.69) заведомо меньше $\varepsilon^{2}$ и вследствие этого может быть отброшен. Таким образом, прояснилась причина справедливости формул (3.64) на отрезке $\delta \leqslant \theta \leqslant \bar{\theta}$. Случай $0 \leqslant \theta \leqslant \delta$ разбирается аналогично с учетом (3.61), (3.66).

Для получения формул (3.65) обратимся ко второму уравнению системы (3.58), считая известной функцию $x_{4}$. Дополняя его при $\theta=0$ начальным условием (3.62) и учитывая свойства (3.64), убеждаемся, что асимптотические представления (3.65) действительно выполняются (неравенство $n_{2} \geqslant 5$ используется для определения порядка остатка во второй формуле в (3.65)). Кроме того, асимптотические равенства (3.65) позволяют придать всем нашим построениям строгий смысл, т. е. убедиться в справедливости априорных оценок (3.67). Действительно, эти оценки заведомо выполняются при любых фиксированных $M_{1}>\max _{w \in \Omega} z_{0}, M_{2}, M_{3}>0$ и при всех достаточно малых $\varepsilon>0$. Лемма доказана. 
На пятом этапе доказательства теоремы 2 сделаем в системе (1.8) замену времени (3.22) и положим в ней

$$
x=\varepsilon^{3} x_{5}, \quad y=\frac{\varepsilon}{\ln (1 / \varepsilon)} y_{5}, \quad z=z_{5}, \quad \tau=\ln \frac{1}{\varepsilon}+\varphi .
$$

В результате получаем систему

$$
\begin{aligned}
\varepsilon \frac{d x_{5}}{d \varphi} & =\left(\varepsilon-z_{5}\right) x_{5}+1, \quad \frac{d y_{5}}{d \varphi}=\left(1-\varepsilon^{2} x_{5}\right) y_{5}+\varepsilon \ln \frac{1}{\varepsilon} \\
\frac{d z_{5}}{d \varphi} & =\left(1-\frac{y_{5}}{\ln (1 / \varepsilon)}\right) z_{5}+\varepsilon^{2}
\end{aligned}
$$

которая при соответствующей циклической перестановке координат переходит в уже изученную систему (3.3). Далее, будем считать, что новое время $\varphi$ из (3.70) меняется на отрезке $\varphi_{1} \leqslant \varphi \leqslant \varphi_{2}$, где

$$
\varphi_{1}=-\left(1-\lambda_{2}\right) \ln \frac{1}{\varepsilon}, \quad \varphi_{2}=\text { const } \in \mathbb{R}
$$

(постоянная $\varphi_{2}$ будет выбрана чуть позже).

Как обычно, дополним систему (3.71) начальными условиями

$$
\left.x_{5}\right|_{\varphi=\varphi_{1}}=x_{5,0}(w, \varepsilon),\left.\quad y_{5}\right|_{\varphi=\varphi_{1}}=y_{5,0}(w, \varepsilon),\left.\quad z_{5}\right|_{\varphi=\varphi_{1}}=z_{5,0}(w, \varepsilon),
$$

заимствованными из предыдущего шага. В силу замен переменных (3.57), (3.70) и формул (3.64)-(3.66) функции $x_{5,0}, y_{5,0}, z_{5,0}$ задаются равенствами

$$
x_{5,0}=\frac{x_{4}(\bar{\theta}, w, \varepsilon)}{\varepsilon^{2}}, \quad y_{5,0}=y_{4}(\bar{\theta}, w, \varepsilon) \varepsilon \ln \frac{1}{\varepsilon}, \quad z_{5,0}=z_{4}(\bar{\theta}, w, \varepsilon) \varepsilon \ln \frac{1}{\varepsilon}
$$

и допускают при $\varepsilon \rightarrow 0$ равномерные по $w \in \Omega$ асимптотики

$$
\begin{gathered}
x_{5,0}=\left(1+O\left(\frac{\ln \ln (1 / \varepsilon)}{\ln (1 / \varepsilon)}\right)\right) \frac{\varepsilon^{\lambda_{2}-1}}{z_{0}}, \quad \frac{\partial x_{5,0}}{\partial y_{0}}=O\left(\varepsilon^{\lambda_{2}} \ln \frac{1}{\varepsilon}\right), \\
\frac{\partial x_{5,0}}{\partial z_{0}}=-\left(1+O\left(\frac{\ln \ln (1 / \varepsilon)}{\ln (1 / \varepsilon)}\right)\right) \frac{\varepsilon^{\lambda_{2}-1}}{z_{0}^{2}} ; \\
y_{5,0}=\left(1+O\left(\frac{\ln \ln (1 / \varepsilon)}{\ln (1 / \varepsilon)}\right)\right) z_{0} \varepsilon^{1-\lambda_{2}}, \quad \frac{\partial y_{5,0}}{\partial y_{0}}=O\left(\frac{\varepsilon^{2-\lambda_{2}} \ln \ln (1 / \varepsilon)}{\ln ^{3}(1 / \varepsilon)}\right), \\
\frac{\partial y_{5,0}}{\partial z_{0}}=\left(1+O\left(\frac{\ln \ln (1 / \varepsilon)}{\ln (1 / \varepsilon)}\right)\right) \varepsilon^{1-\lambda_{2}} ; \\
\left.z_{5,0}=\left(1+\frac{\ln \ln (1 / \varepsilon)}{\ln (1 / \varepsilon)}\right)\right) z_{0} \varepsilon^{1-\lambda_{2}}, \frac{\partial z_{5,0}}{\partial y_{0}}=O\left(\varepsilon^{2-\lambda_{2}} \ln \frac{1}{\varepsilon}\right), \\
\frac{\partial z_{5,0}}{\partial z_{0}}=\left(1+O\left(\frac{\ln \ln (1 / \varepsilon)}{\ln (1 / \varepsilon)}\right)\right) \varepsilon^{1-\lambda_{2}} .
\end{gathered}
$$

Обозначим через $x_{5}(\varphi, w, \varepsilon), y_{5}(\varphi, w, \varepsilon), z_{5}(\varphi, w, \varepsilon)$ компоненты решения задачи Коши (3.71), (3.73). Справедливо следующее утверждение. 
Лемма 5. На отрезке $\varphi_{1} \leqslant \varphi \leqslant \varphi_{2}$ для функиий $y_{5}(\varphi, w, \varepsilon), z_{5}(\varphi, w, \varepsilon)$ при $\varepsilon \rightarrow 0$ имеют место равномерные по $\varphi, w$ асимптотические представления

$$
\begin{aligned}
y_{5} & =\left(1+O\left(\frac{\ln \ln (1 / \varepsilon)}{\ln (1 / \varepsilon)}\right)\right) z_{0} e^{\varphi}, & & \frac{\partial y_{5}}{\partial \varphi}=\left(1+O\left(\frac{\ln \ln (1 / \varepsilon)}{\ln (1 / \varepsilon)}\right)\right) z_{0} e^{\varphi}, \\
\frac{\partial y_{5}}{\partial y_{0}} & =O\left(\frac{\varepsilon \ln \ln (1 / \varepsilon)}{\ln ^{3}(1 / \varepsilon)}\right) e^{\varphi}, & \frac{\partial y_{5}}{\partial z_{0}} & =\left(1+O\left(\frac{\ln \ln (1 / \varepsilon)}{\ln (1 / \varepsilon)}\right)\right) e^{\varphi} \\
z_{5} & =\left(1+O\left(\frac{\ln \ln (1 / \varepsilon)}{\ln (1 / \varepsilon)}\right)\right) z_{0} e^{\varphi}, & \frac{\partial z_{5}}{\partial \varphi} & =\left(1+O\left(\frac{\ln \ln (1 / \varepsilon)}{\ln (1 / \varepsilon)}\right)\right) z_{0} e^{\varphi}, \\
\frac{\partial z_{5}}{\partial y_{0}} & =O\left(\varepsilon \ln \frac{1}{\varepsilon}\right) e^{\varphi}, & \frac{\partial z_{5}}{\partial z_{0}} & =\left(1+O\left(\frac{\ln \ln (1 / \varepsilon)}{\ln (1 / \varepsilon)}\right)\right) e^{\varphi} .
\end{aligned}
$$

В случае функиии $x_{5}(\varphi, w, \varepsilon)$ соответствующие асимптотические представления

$$
\begin{aligned}
x_{5} & =\left(1+O\left(\frac{\ln \ln (1 / \varepsilon)}{\ln (1 / \varepsilon)}\right)\right) \frac{e^{-\varphi}}{z_{0}}, \quad \frac{\partial x_{5}}{\partial y_{0}}=O\left(\varepsilon \ln \frac{1}{\varepsilon}\right) e^{-\varphi}, \\
\frac{\partial x_{5}}{\partial \varphi} & =-\left(1+O\left(\frac{\ln \ln (1 / \varepsilon)}{\ln (1 / \varepsilon)}\right)\right) \frac{e^{-\varphi}}{z_{0}}, \\
\frac{\partial x_{5}}{\partial z_{0}} & =-\left(1+O\left(\frac{\ln \ln (1 / \varepsilon)}{\ln (1 / \varepsilon)}\right)\right) \frac{e^{-\varphi}}{z_{0}^{2}}
\end{aligned}
$$

выполняются при $\varepsilon \rightarrow 0$ равномерно по $\varphi \in\left[\varphi_{1}+\delta, \varphi_{2}\right], w \in \Omega$, где $\delta-$ произвольно фиксированная положительная постоянная.

Доказательство леммы 5 с незначительными изменениями повторяет доказательство леммы 1, так как система (3.3) переходит в (3.71) при замене

$$
\left(x_{1}, y_{1}, z_{1}\right) \rightarrow\left(z_{5}, x_{5}, y_{5}\right)
$$

и при переобозначении $t \rightarrow \varphi$. Поэтому акцентируем внимание лишь на некоторых новых моментах.

Сначала, отталкиваясь от априорных оценок

$$
x_{5} \leqslant M_{1} e^{-\varphi}, \quad\left|\frac{\partial x_{5}}{\partial y_{0}}\right| \leqslant M_{2}, \quad\left|\frac{\partial x_{5}}{\partial z_{0}}\right| \leqslant M_{3} e^{-\varphi},
$$

последовательно выводим группы формул (3.77) и (3.78). Далее, привлечем устойчивое многообразие медленных движений

$$
\left\{\left(x_{5}, y_{5}, z_{5}\right): x_{5}=H\left(z_{5}, y_{5}, \varepsilon\right)\right\}
$$

получающееся из (3.16) при замене (3.80). Единственное отличие от (3.16) состоит в том, что здесь переменные $\left(z_{5}, y_{5}\right)$ пробегают множество вида

$$
\left\{\left(z_{5}, y_{5}\right): M_{4} \varepsilon^{1-\lambda_{2}} \leqslant z_{5} \leqslant M_{5},\left|y_{5}\right| \leqslant M_{6}\right\}, \quad M_{4}, M_{5}, M_{6}=\text { const }>0 .
$$

Что же касается асимптотических представлений для $H, \partial H / \partial z_{5}, \partial H / \partial y_{5}$, то они получаются из (3.17)-(3.19) при замене (3.80). 
Как и при доказательстве леммы 1, наличие многообразия (3.82), (3.83) позволяет не только установить асимптотические представления (3.79) на любом отрезке вида $\varphi_{1}+\delta \leqslant \varphi \leqslant \varphi_{2}, \delta=$ const $>0$, но и убедиться в справедливости априорных предположений (3.81). Соответствующие детали опустим.

Вернемся к доказательству теоремы 2. Проанализируем аналогичное (2.3) уравнение

$$
z_{5}(\varphi, w, \varepsilon)=1
$$

Согласно (3.78) при $\varepsilon \rightarrow 0$ оно переходит в уравнение $z_{0} e^{\varphi}=1$, имеющее единственный корень $\varphi=\ln \left(1 / z_{0}\right)$. Далее, предположим, что постоянная $\varphi_{2}$ из $(3.72)$, которую до этого мы считали произвольной, удовлетворяет условию $\varphi_{2}>\max _{w \in \Omega} \ln \left(1 / z_{0}\right)$. Тогда снова обращаясь к равенствам (3.78), убеждаемся, что в этом случае уравнение (3.84) имеет на отрезке $\varphi_{1} \leqslant \varphi \leqslant \varphi_{2}$ единственный корень $\varphi=\varphi_{0}(w, \varepsilon)$, допускающий при $\varepsilon \rightarrow 0$ равномерную по $w \in \Omega$ асимптотику

$$
\begin{gathered}
\varphi_{0}=\ln \frac{1}{z_{0}}+O\left(\frac{\ln \ln (1 / \varepsilon)}{\ln (1 / \varepsilon)}\right), \quad \frac{\partial \varphi_{0}}{\partial y_{0}}=O\left(\varepsilon \ln \frac{1}{\varepsilon}\right), \\
\frac{\partial \varphi_{0}}{\partial z_{0}}=-\frac{1}{z_{0}}+O\left(\frac{\ln \ln (1 / \varepsilon)}{\ln (1 / \varepsilon)}\right) .
\end{gathered}
$$

Наличие корня (3.85) означает, что на множестве $\Omega$ корректно определен оператор (2.4). Действительно, в силу замен (3.22), (3.70) фигурирующий в (2.4) момент времени $t=h(w, \varepsilon)$ задается равенством

$$
h(w, \varepsilon)=\ln \frac{1}{\varepsilon}+\ln \ln \frac{1}{\varepsilon}+\varphi_{0}(w, \varepsilon) .
$$

Что же касается оператора $\Pi_{0}$, то согласно леммам 1-5 для него справедливо представление

$$
\Pi_{0}(w, \varepsilon)=\left.\left(x_{5}(\varphi, w, \varepsilon), y_{5}(\varphi, w, \varepsilon)\right)\right|_{\varphi=\varphi_{0}(w, \varepsilon)} .
$$

Отсюда и из соотношений (3.77), (3.79), (3.85) вытекает асимптотическое равенство (2.6). Теорема 2 доказана.

\section{4. ЗАКЛЮЧЕНИЕ}

Прежде всего завершим доказательство теоремы 1. Напомним, что нам осталось показать справедливость асимптотических равенств (1.10). В связи с этим обратим внимание, что в силу $(3.77),(3.79),(3.85)$ неподвижная точка $w=w_{*}(\varepsilon)$ оператора (3.87) допускает при $\varepsilon \rightarrow 0$ асимптотику

$$
w_{*}(\varepsilon)=w_{*}+O\left(\frac{\ln \ln (1 / \varepsilon)}{\ln (1 / \varepsilon)}\right) .
$$

Заметим далее, что первое соотношение (1.10) вытекает из формул $(3.23),(3.32)$ при $w=w_{*}(\varepsilon)$ и из (4.1). Что касается минимума $x_{*}(t, \varepsilon)$, то согласно свойству самосимметричности цикла (1.9) он определяется как минимальное значение из $\min x$, $\min y, \min z$. Из лемм $1-5$ следует, что это значение имеет порядок $\varepsilon^{3} / \ln (1 / \varepsilon)$. Точнее говоря, в силу (3.34), (4.1) для него справедливо асимптотическое равенство 
из (1.10). Остается заметить, что последняя формула в (1.10) - следствие соотношений (3.85), (3.86), (4.1). Теорема 1 полностью доказана.

Для получения более наглядного представления о свойствах цикла (1.9) дадим качественное описание эволюции во времени его компоненты $x_{*}(t, \varepsilon)$ при $t \geqslant 0$. Из лемм 1-5 вытекают следующие факты. Сначала $x_{*}(t, \varepsilon)$ монотонно возрастает примерно по экспоненциальному закону $x_{*}=e^{t}$ и достигает за время порядка $\ln \ln (1 / \varepsilon)$ значений порядка $\ln (1 / \varepsilon)$. Далее следует всплеск переменной $x_{*}(t, \varepsilon)$, происходящий по закону

$$
\left.x_{*}(t, \varepsilon) \sim \ln \frac{1}{\varepsilon} \cdot \exp \left(\tau-e^{\tau}\right)\right|_{\tau=t-\ln \ln (1 / \varepsilon)} .
$$

Этот всплеск сменяется спадом до асимптотически малых значений, который длится время порядка $\ln \ln (1 / \varepsilon)$. Затем в течение промежутка времени порядка $\ln (1 / \varepsilon)$ компонента $x_{*}(t, \varepsilon)$ асимптотически мала. И, наконец, происходит экспоненциальный подъем $x_{*}(t, \varepsilon)$ от асимптотически малых значений до значений порядка единицы, который также занимает время порядка $\ln (1 / \varepsilon)$. После этого весь процесс повторяется.

Следует также отметить, что всплески компонент цикла (1.9) разделены во времени, т. е. при каждом $t$ лишь одна компонента имеет порядок единицы или асимптотически велика, а две остальные асимптотически малы. В этом, собственно, и состоит биологический механизм репрессии: в любой момент времени какой-либо один ген подавляет два других. Наша модель (1.6) дает математическое описание указанного механизма.

В заключение отметим, что предложенный в данной статье новый метод математического моделирования искусственных генных сетей может быть применен не только к простейшей сети, показанной на рис. 1, но и к более сложным сетям. В самом общем случае для так называемой полносвязной генной сети наш подход приводит к аналогичной (1.4) системе с запаздываниями

$$
\begin{aligned}
& \dot{m}_{j}=\frac{r_{1, j}}{1+\sum_{k=1}^{n} a_{j, k}}\left[1+\sum_{k=1}^{n} a_{j, k}\left(1-u_{k}\right)-m_{j}\left(t-h_{1, j}\right)\right] m_{j}+\alpha_{j}, \\
& \dot{u}_{j}=r_{2, j}\left[\sum_{k=1}^{n} \delta_{j, k} m_{k}-u_{j}\left(t-h_{2, j}\right)\right] u_{j}, \quad j=1,2, \ldots, n,
\end{aligned}
$$

где все параметры $r_{1, j}, r_{2, j}, a_{j, k}, h_{1, j}, h_{2, j}, \delta_{j, k}, \alpha_{j}$ неотрицательны и

$$
r_{1, j}>0, \quad r_{2, j}>0, \quad \sum_{k=1}^{n} \delta_{j, k}=1 .
$$

В пояснении здесь нуждается лишь третье соотношение. В экологической интерпретации коэффициент $\delta_{j, k}-$ это доля $k$-й жертвы в рационе $j$-го хищника. Тем самым при любом $j$ сумма $\delta_{j, k}$ по $k$ должна равняться единице.

Изучение системы $(4.2),(4.3)$ целесообразно начать с $n=1$, когда имеется один ген-авторепрессор. В этом случае указанная система принимает вид

$$
\dot{m}=\frac{r_{1}}{1+a}\left[1+a(1-u)-m\left(t-h_{1}\right)\right] m+\alpha, \quad \dot{u}=r_{2}\left[m-u\left(t-h_{2}\right)\right] u,
$$


где $r_{1}, r_{2}>0, a \geqslant 0, h_{1}, h_{2} \geqslant 0, \alpha \geqslant 0$. Далее, предположим, что $h_{1}>0, h_{2}=0$ (без ограничения общности можно считать, что $\left.h_{1}=1\right), r_{1}=r \sim 1, r_{2} \gg 1$. Тогда, опираясь на уже неоднократно применявшийся принцип сведения [2], имеем $m=u$. Для концентрации белка $u(t)$ из (4.5) получается уравнение

$$
\dot{u}=\frac{r}{1+a}[1+a(1-u)-u(t-1)] u+\alpha .
$$

При $\alpha=0, a=\mathrm{const} \in(0,1), r \gg 1$ уравнение (4.6) представляет собой математическую модель популяции млекопитающих с учетом каннибализма. В этом случае вопросы о существовании, асимптотике и устойчивости релаксационного цикла уравнения (4.6) изучены в монографиях [9], [11]. В случае же, когда $\alpha=$ const $>0$, $a=$ const $\geqslant 0, r \gg 1$, эти проблемы пока не решены.

\section{Список литературы}

[1] M. B. Elowitz, S. Leibler, "A synthetic oscillatory network of transcriptional regulators", Nature, 403 (2000), 335-338.

[2] А.Н. Тихонов, "Системы дифференциальных уравнений, содержащие малые параметры при производных", Матем. сб., 31(73):3 (1952), 575-586.

[3] Е. П. Волокитин, "О предельных циклах в простейшей модели гипотетической генной сети", Сиб. журн. индустр. матем., 7:3 (2004), 57-65.

[4] O. Buşe, A. Kuznetsov, R. A. Pérez, "Existence of limit cycles in the repressilator equations", Internat. J. Bifurcation Chaos, 19:12 (2009), 4097-4106.

[5] O. Buşe, R. Pérez, A. Kuznetsov, "Dynamical properties of the repressilator model", Phys. Rev. E, 81:6 (2010), 066206, 7 pp.

[6] В.А. Лихошвай, Ю. Г. Матушкин, С. И. Фадеев, “Задачи теории функционирования генных сетей", Сиб. журн. индустр. матем., 6:2 (2003), 64-80.

[7] Г. В. Демиденко, Н. А. Колчанов, В. А. Лихошвай, Ю. Г. Матушкин, С. И. Фадеев, "Математическое моделирование регулярных контуров генных сетей", Журн. вычисл. матем. и матем. физ., 44:12 (2004), 2276-2295.

[8] С. И. Фадеев, В. А. Лихошвай, "О гипотетических генных сетях", Сиб. журн. индустр. матем., 6:3 (2003), 134-153.

[9] А. Ю. Колесов, Ю. С. Колесов, Релаксачионные колебания в математических моделях экологии, Тр. МИАН, 199, ред. Е. Ф. Мищенко, Наука, М., 1993.

[10] А.Б. Васильева, В.Ф. Бутузов, Асимптотические разложения решений сингулярно возмущенных уравнений, Наука, М., 1973.

[11] Е.Ф. Мищенко, В. А. Садовничий, А. Ю. Колесов, Н. Х. Розов, Многоликий хаос, Физматлит, М., 2012. 